\title{
Radiation-Driven Warping: The Origin of Warps and Precession in Accretion Disks
}

\author{
Philip R. Maloney \\ Center for Astrophysics and Space Astronomy, University of Colorado, \\ Boulder, CO 80309-0389
}

\author{
Mitchell C. Begelman ${ }^{1}$ \\ JILA, University of Colorado, Boulder, CO 80309-0440
}

\begin{abstract}
.
A geometrically thin, optically thick, warped accretion disk with a central source of luminosity is subject to non-axisymmetric forces due to radiation pressure; the resulting torque acts to modify the warp. Initially planar accretion disks are unstable to warping driven by radiation torque, as shown in a local analysis by Pringle (1996) and a global analysis of the stable and unstable modes by Maloney, Begelman, \& Pringle (1996). In general, the warp also precesses.

We discuss the nature of this instability, and its possible implications for accretion disks in X-ray binaries and active galactic nuclei. Specifically, we argue that this effect provides a plausible explanation for the misalignment and precession of the accretion disks in X-ray binaries such as SS 433 and Her X-1; the same mechanism explains why the maser disk in NGC 4258 is warped.
\end{abstract}

\section{Introduction}

For more than two decades, observational evidence has accumulated for the presence of tilted, precessing accretion disks in X-ray binary systems. The initial discovery of a 35-day period in the X-ray flux from Her X-1 (Tananbaum et al. 1972) was interpreted almost immediately as the result of periodic obscuration by a precessing accretion disk which is tilted with respect to the plane of the binary system (Katz 1973). Katz proposed that the precession was forced by the torque from the companion star, but left unexplained the origin of the disk's misalignment. (The fact that the optical light from the companion star HZ Her - which is largely reprocessed X-ray flux - does not vary significantly over the 35-day period indicates that the intrinsic X-ray luminosity is not varying.) An alternative possibility was proposed by Roberts (1974), who suggested that it was actually the companion star that was misaligned and precessing; the mate-

\footnotetext{
${ }^{1}$ Also at Department of Astrophysical, Planetary, and Atmospheric Sciences, University of Colorado, Boulder.
} 
rial in the accretion disk would be "slaved" to the precession of the companion star provided the residence time in the disk was sufficiently short.

Interest in tilted, precessing accretion disks was greatly stimulated by the discovery of the precessing relativistic jets in SS 433 (for a review see Margon 1984). The systematic velocity variations of the high-velocity lines, the morphology of the radio jets, and optical photometry of the system all indicate that the accretion disk is precessing with a period of 164 days; both the inner region of the disk, near the compact object (to account for the jet precession) and the outer edge (to account for the optical photometry) must precess at the same rate, indicating global precession of the disk. Global precession of the disk is also indicated for Her X-1, to explain the systematic variation of the pulse profiles over the 35-day cycle (Trümper et al. 1986; Petterson, Rothschild, \& Gruber 1991).

A number of other X-ray binaries, both high and low mass, show evidence for long period variations and precessing, inclined accretion disks: LMC X-4 (30.5 days), Cyg X-1 (294 days), XB1820-303 (175 days), LMC X-3 (198 or 99 days), and Cyg X-2 (77 days) (Priedhorsky \& Holt 1987, and references therein; Cowley et al. 1991; Smale \& Lochner 1992; White, Nagase, \& Parmar 1995 , and references therein). Given the small number of X-ray binaries for which adequate data exist to test for the existence of such periodicities, it is evident that precessing, inclined accretion disks are in fact common in X-ray binaries. Further evidence for the existence of non-planar accretion disks, in a very different context, is provided by the extraordinary warped disk of water masers in NGC 4258 (Miyoshi et al. 1995; Herrnstein, Greenhill, \& Moran 1996).

In spite of twenty years of theoretical work, however, there is no accepted model for producing and maintaining a steadily precessing, tilted accretion disk. The initial models for Her X-1 both suffer from severe flaws. The model of Katz (1973) requires that the outer disk be tilted with respect to the binary plane, but has no mechanism for producing such a tilt; furthermore, the strong $\left(\propto r^{3 / 2}\right)$ radial dependence of the quadrupole forcing term implies that the precession can only be driven at large radius, and the free precession timescale at the outer edge of the disk is too short compared to the observed period. This shortcoming was overcome by Merritt \& Petterson (1980), who included the effects of viscous torques in the disk and showed that it was possible to obtain global modes with precession rates slower than the free precession time at the outer edge. However, the tilt of the disk at the outer edge was still simply imposed as a boundary condition, with no physical explanation. The slaved disk model of Roberts (1974) requires that the rotation axis of the companion star be inclined to the binary plane; however, the axial tilt will decay by tidal damping faster than the orbit will circularize (Chevalier 1976; Papaloizou \& Pringle 1982), and the observed periods are too short compared to the expected periods for synchronously rotating binary systems (Hut \& van den Heuvel 1981). Furthermore, precession of the companion star is expected to produce a more precise clock than is seen in Her X-1, for which the inferred precession period varies by up to ten percent (Boynton, Crosa, \& Deeter 1980). Other suggested mechanisms, such as precession of an oblate neutron star (Brecher 1972) also suffer from the fact that, at least for Her X-1 and SS 433, a global precession 


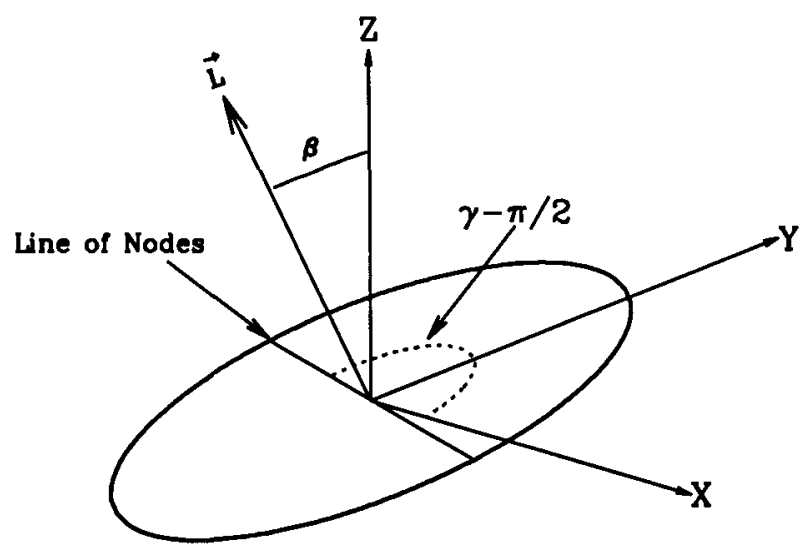

Figure 1. Geometry of a tilted annulus of an accretion disk. The equatorial plane of the binary system is the $X Y$ plane; the ring is tipped by an angle $\beta$ relative to the normal to the equatorial plane (the $Z$ - axis). Also marked is the line of nodes; the descending node makes an angle of $\gamma-\pi / 2$ with the $X$-axis. The angular momentum vector of the ring is $\overrightarrow{\mathrm{L}}$.

mode is required, and it is very difficult to communicate a fixed precession rate through a viscous, differentially-rotating disk.

\section{Radiation-Driven Warping}

Fortunately, there is a natural mechanism for producing warping and global precessing modes in centrally-irradiated accretion disks: namely, warping of disks due to radiation pressure from the central source.

Consider an annulus of an accretion disk with a central source of luminosity (Figure 1). If we warp this annulus slightly, it will see the central source of radiation, and, if the disk is optically thick, it will absorb the incident flux. The momentum associated with this absorbed incident flux is purely radial, and so the force due to the incident radiation will produce zero torque. If the disk is optically thick to re-emission of the absorbed radiation, however, the net reemitted flux will be normal to the local plane of the disk. The radiation pressure acting on the irradiated surface due to the re-emitted radiation will therefore exert a torque at each exposed point of the annulus. If the annulus is warped, i.e., the local angle of tilt varies with position, then the net torque on the ring will be non-zero, because the deposition of radiation momentum per unit area of the ring will be non-axisymmetric. In general, the net torque will be non-zero provided that there is a radial gradient in either the tilt angle $\beta$ (in which case the ring will precess) or the angle of the line of nodes (in which case the torque will cause the tilt to change); see Figure 1 . Note that the disk must be optically thick both to absorption of the incident radiation and to re-radiation of the 
absorbed flux. If the disk is optically thin to absorption, then the absorption rate will be essentially constant through the annulus, and the flux re-radiated from the two faces of the annulus (the irradiated face and the opposite face, which does not see the central source directly) will be the same, so that the re-emitted radiation will exert zero torque. If the disk is optically thin to reemission, then the re-emitted radiation will be isotropic, and again the torque will be zero.

The importance of radiation pressure for altering the shape of a priori warped accretion disks was first pointed out by Petterson (1977). Detailed numerical models of warped disks were constructed by Iping \& Petterson (1990), while disk models including a related effect - the torque from a non-axisymmetric wind driven off the surface of a disk by X-ray heating - were presented by Schandl \& Meyer (1994). Iping \& Petterson, in particular, suggested that radiation pressure forces might be very important in establishing the shape of the disk and the precession rate.

This hint that accretion disks might actually be unstable to warping due to radiation pressure was confirmed by Pringle (1996), who showed via a local (WKB) analysis that isothermal $\alpha$-disks (radial surface density $\Sigma \propto R^{-3 / 2}$ ), even if initially planar, are unstable to radiation-pressure warping. However, a short-wavelength WKB analysis is only marginally justified, as the unstable modes in Pringle's analysis have wavenumber $k \sim 2 \pi / R$. Furthermore, it is impossible to specify the boundary conditions in a purely local analysis, and it turns out that the boundary conditions play a crucial role.

Maloney, Begelman, \& Pringle (1996) extended Pringle's work in a global analysis, and solved the isothermal case analytically. For the inner boundary condition we require that no viscous torque be exerted on the disk at the origin; this requires that the local angle of tilt $\beta \rightarrow$ constant as $R \rightarrow 0$, so that the disk becomes asymptotically flat (but not necessarily untilted) at small radii. The outer boundary condition is that $\beta \rightarrow 0$ at some finite radius. Physically, this outer boundary condition is required because the disk material is supplied in the equatorial plane at the disk's outer edge; therefore, whatever the actual shape of the warped disk, it must return to the equatorial plane of the system at the accretion disk boundary.

There are stable, unstable, and time-independent modes for warping of the disk. The precession rate of the warp is in general non-zero. There is only a single value of the precession rate (i.e., the real part of the frequency eigenvalue) for which the warp of the disk returns to the equatorial plane at any radius; this is the only solution that can be matched to a disk that is unwarped at its outer boundary. The growth rate of the warp (the imaginary part of the eigenvalue) can take on a range of values, depending on the outer boundary condition. The location of the warp and the first zero move outward with increasing growth rate (Figure 2), so that the warp grows from the "outside" in. The warp precesses as a fixed pattern by twisting the disk in such a way that the radiation torque causes each radial annulus to precess at the same rate. Figure 3 shows a surface plot of the steady-state mode of the isothermal disk, plotted out to the radius at which the warp returns to the equatorial plane. The amplitude of the warp has been fixed at $10 \%$ of the outer radius. 


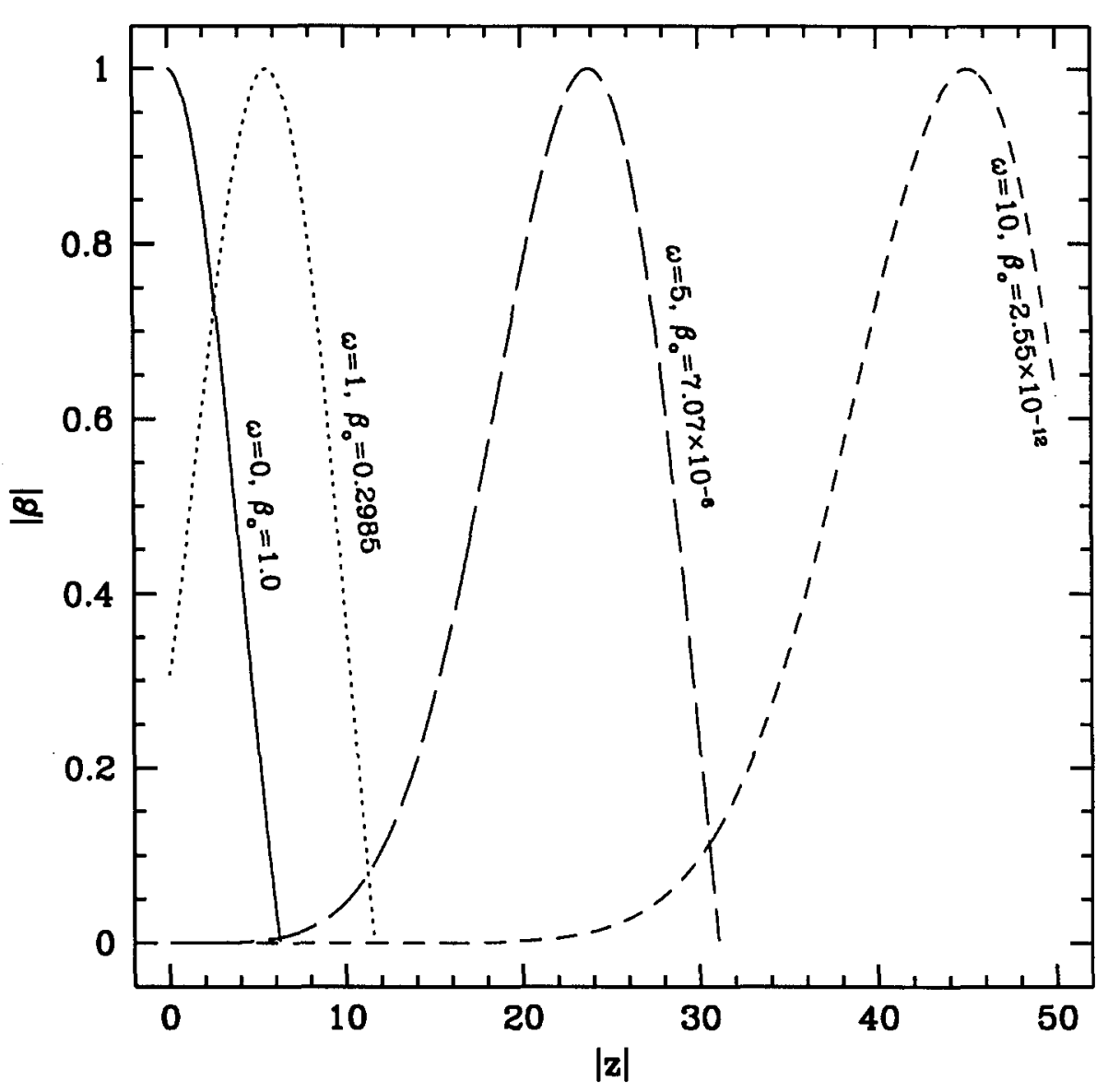

Figure 2. The amplitude of the local angle of tilt $|\beta|$ for several of the growing solutions of the twist equation (for an isothermal disk) is plotted against the radius variable $|z|$, which is proportional to the radiative efficiency $\epsilon$ times $\left(R / R_{s}\right)^{1 / 2}$, where $R_{s}$ is the Schwarzschild radius. For clarity the solutions have been plotted only to the location of the first zero for each mode. All the modes have the same precession rate and grow as $e^{\omega t}$. They have all been normalized so that the maximum amplitude is 1 ; each curve is labeled with the dimensionless growth rate $\omega$ and with the normalization constant $\beta_{o}$, i.e., the magnitude of the tilt at the origin. For $\omega \gg 1$ this is extremely small, so that for modes with high growth rates the disks remain flat interior to a critical radius. The maximum value of $\omega$ for a disk is fixed by the largest unstable radius, which is either the physical edge of the disk or the radius at which it becomes optically thin. 


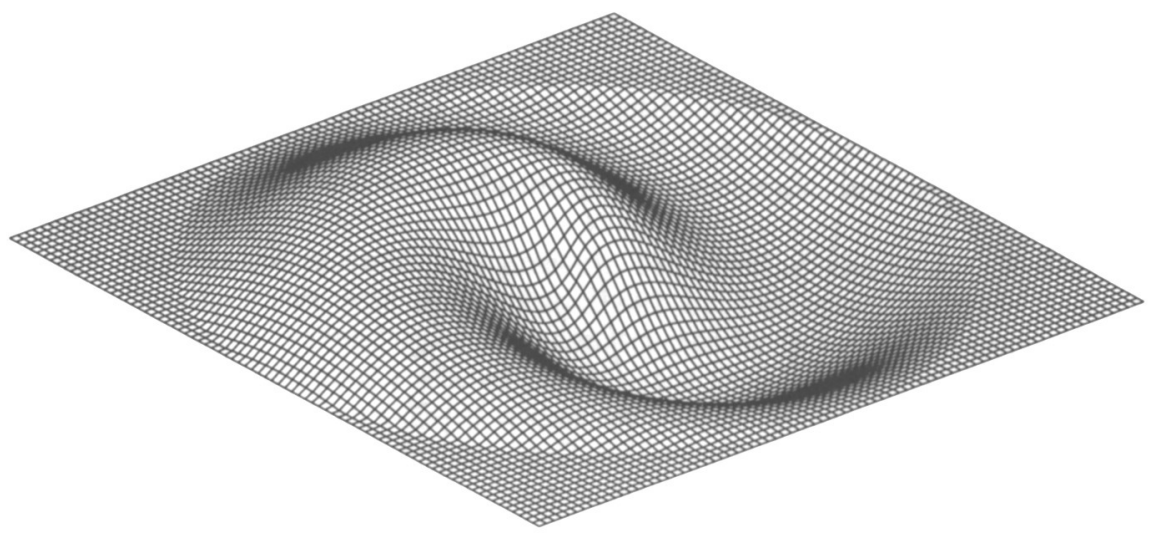

Figure 3. Surface plot of the warp shape for the steady-state mode of an isothermal $(\delta=3 / 2)$ accretion disk. The warp has been plotted out to the radius at which it returns to the equatorial plane; the amplitude of the warp has been fixed at $10 \%$ of this radius.

The isothermal case is actually degenerate (the real parts of all the eigenvalues are identical), but the degeneracy is lifted for nonisothermal surface density distributions, and a complex pattern of modes results (Maloney, Begelman, \& Nowak 1996). However, there are a number of features of the instability which are generic:

- The radiation torque per unit area is $\Gamma \sim\left(L / 4 \pi R^{2} c\right) \times R$, while the angular momentum density is $l \sim \Omega R^{2} \Sigma \sim \Omega R^{2} \dot{M} / \nu$, where $\Omega$ is the angular velocity at radius $R$ in the disk, $\dot{M}$ is the mass accretion rate, and $\nu$ is the kinematic viscosity. The radiation torque timescale is thus given by

$$
t_{\mathrm{rad}} \sim \frac{\Omega R^{3}}{c \nu} \frac{\dot{M} c^{2}}{L}=\frac{\Omega R^{3}}{c \nu} \epsilon^{-1}
$$

which, for an accretion-fueled source, depends only on the accretion efficiency $\epsilon=L / \dot{M} c^{2}$ and not on the luminosity $L$ and the mass accretion rate individually.

- The viscous timescale is $t_{\text {visc }} \sim R^{2} / \nu$, so the ratio of viscous to radiation torque timescales is given by

$$
\frac{t_{\mathrm{visc}}}{t_{\mathrm{rad}}} \sim \frac{\epsilon c}{\Omega R} \sim \epsilon\left(\frac{R}{R_{g}}\right)^{1 / 2} ; \quad R_{g}=\frac{G M}{c^{2}}
$$

which depends only on $\epsilon$ and the radius normalized to the gravitational radius. Thus the radiation torque always wins out at large radius, i.e., for $R>\epsilon^{-2} R_{g}$, but viscosity always dominates near the center. This is why the disk will be flat (but in general will have non-zero tilt) at small radius. 
- For a fixed outer boundary radius, there is a single value of the precession rate; the location of the warp always moves outward in radius with increasing growth rate. This is why the outer boundary condition is crucial: the outer boundary radius determines the growth rate of the warp. This selfregulation means that the growth time will be of approximately the same order as the precession time. For X-ray binaries, this timescale is much shorter than the lifetime of the accretion disks, and so the warps in X-ray binary systems presumably reach the steady-state solutions. Although the inner region of the disk $\left(R \ll \epsilon^{-2} R_{g}\right)$ will be flat, it will generally be tilted with respect to the binary orbit plane, and the entire disk shape will precess globally, at the same rate. Because it is set by conditions in the disk, the precession timescale may not be precisely constant with time, but will be coupled to variations in the outer boundary radius, the disk surface density distribution, and the accretion efficiency.

\section{Applications}

\subsection{X-ray Binaries}

The precession timescale due to radiation pressure can be written as

$$
t_{\text {prec }} \sim 32\left(\frac{\alpha}{0.1}\right)^{-1}\left(\frac{\epsilon}{0.1}\right)^{-1}\left(\frac{h / R}{0.01}\right)^{-2} R_{11} \text { days }
$$

where $\alpha$ is the usual disk viscosity parameter, $\epsilon=L / \dot{M} c^{2}$ is the radiative efficiency (assuming an accretion-fueled source), $h / R$ is the thickness/radius ratio of the disk at the location of the warp, and the outer radius of the disk is at $R=10^{11} R_{11} \mathrm{~cm}$; we have normalized to typical values for an $\mathrm{X}$-ray binary. This is clearly of the right order to explain the observed precession periods.

In X-ray binaries, however, the quadrupole torque due to the companion star can also have a significant influence on the warp and its precession rate (e.g., Merritt \& Petterson 1980). In the absence of such a torque, the precession of the warp can be either prograde or retrograde with respect to the direction of rotation, due to the antisymmetry of the net torque direction for inversion of the warp through the origin. The torque due to the other component of the binary breaks this symmetry, however, and establishes a preferred direction for precession, counter to the orbital direction. The radiation-driven warping mechanism is still essential in this case because it establishes the warp on which the quadrupole torque operates. Because of the strong radial dependence of the quadrupole torque term, there will be a characteristic radius at which the radiation torque and quadrupole torque will be equal, with the quadrupole term rapidly dominating at larger $R$ and the radiation term at smaller $R$. Because of this dominance at large $R$, if the quadrupole term is important it will always set the boundary condition at the outer edge of the disk, thereby controlling the resulting precession rate.

\subsection{The Warped Maser Disk of NGC 4258}

VLBI observations of the $22 \mathrm{GHz}$ water maser line emission from the nucleus of NGC 4258 (which possesses a low-luminosity active nucleus) have shown that the 
maser emission arises in a thin, warped disk, with an inner radius of $0.13 \mathrm{pc}$ and an outer radius of $0.25 \mathrm{pc}$, which exhibits a perfectly Keplerian velocity curve to within the measurement errors (Miyoshi et al. 1995; Greenhill et al. 1995). The derived central mass is $M_{c}=3.6 \times 10^{7} M_{\odot}$, and dynamical arguments essentially rule out mass models other than a supermassive black hole (Maoz 1995). The obliquity of the warp is $\mu \approx 0.25$ at the outer edge of the masing zone ( $L$. Greenhill 1995, private communication) and decreases steadily inward.

Neufeld, Maloney, \& Conger (1994) showed that X-ray irradiation of dense molecular gas is an effective mechanism for generating powerful water maser emission. By modeling the NGC 4258 disk using the standard $\alpha$-prescription for viscosity, Neufeld \& Maloney (1995) showed that the disk would be extremely thin $\left(h / R \ll 10^{-2}\right)$, that the transition from molecular to atomic gas should be identified with the outer edge of the masing region, and that the mass accretion rate through the disk is $\dot{M} / \alpha \approx 7 \times 10^{-5} M_{\odot} \mathrm{yr}^{-1}$. (See also Maloney, this volume.) This mass accretion rate in turn implies that the radiative efficiency $\epsilon \approx 0.1$. The existence of the warp in this picture is crucial, since for a flat disk neither the greatly reduced irradiation from the central source nor heating by viscous dissipation would keep the disk warm enough to produce substantial amounts of water or excite the masing transitions. In fact, Neufeld \& Maloney (1995) suggested that the inner edge of the masing disk marked the inner boundary of the warp, as is the case in the model for the warp derived by Miyoshi et al. (1995). Although an arbitrary imposed warp would be kinematically stable in a Keplerian potential, since the vertical and azimuthal frequencies are the same, the origin of the warp in NGC 4258 remained puzzling.

The radiation-driven warping instability provides a plausible explanation for the warping of the disk (Maloney, Begelman, \& Pringle 1996). The isothermal models depicted in Figure 2 are applicable to this case and, given the estimated locattion of the warp and the accretion parameters derived by Neufeld \& Maloney (1995), we estimate the dimensionless growth rate to be $\omega \approx 10$. It is likely that $\omega_{\min }$ is not much smaller than this value, due to the limit set by the finite age of the accretion disk. The characteristic timescale for growth of the instability is

$$
\tau_{i}(\omega) \sim \frac{4}{\omega} \frac{c R_{s}}{\alpha c_{s}^{2} \epsilon} \approx 3 \times 10^{7} \frac{M_{8}}{\omega \alpha T_{3} \epsilon_{0.1}} \mathrm{yrs}
$$

where $M_{c}=10^{8} M_{8} M_{\odot}, c_{s}$ is the sound speed, the gas temperature $T=10^{3} T_{3}$ $\mathrm{K}$, and the numerical coefficient is for molecular gas. In the masing molecular zone, $0.3<T_{3}<1$. While the characteristic growth time for the $\omega=10$ mode in NGC 4258 is $\tau_{i} \sim 5 \times 10^{6}$ years, the time required for even the $\omega=5$ mode to become comparable in amplitude is an order of magnitude longer. Thus we expect that the warp will be dominated by modes with $\omega$ of order 10 , unless the accretion disk is extremely long-lived ( $\tau_{\text {disk }} \gg 10^{8}$ years). In contrast to the $\mathrm{X}$-ray binaries, the warp in NGC 4258 is far from attaining a steady state.

Note that the predictions of the radiation-driven warp instability model are in reasonable agreement with the observations of NGC 4258 , provided that $\epsilon \sim 0.1$. This instability cannot provide an explanation for the warp if NGC 4258 possesses an advection-dominated accretion disk with $\epsilon \sim 10^{-3}$, as suggested by Lasota et al. (1996). This would require $\omega<1$, implying a prohibitively long growth timescale, with $\tau_{i}>10^{9}$ years. 


\section{Conclusions}

Warping and precession driven by radiation pressure offers a robust mechanism for producing tilted, precessing accretion disks in accreting binary systems such as Her X-1 and SS 433, and warped disks in AGNs. Because the warp adjusts itself in such a way as to precess at a uniform rate at all radii, with the rate fixed by the outer boundary condition, radiation-driven warping is an inherently global mechanism, thereby avoiding the difficulties inherent in other proposed mechanisms for producing warping and precession. This mechanism can thus explain the simultaneous precession of inner disks (as evidenced by the jets of SS 433 and the pulse profile variations of Her X-1) and outer disks (as required to match the periodicities in X-ray flux and disk emission in these same objects).

Our work to date has been based on a linearized version of the equations governing the evolution of a twisted, viscous accretion disk (e.g., Pringle 1992). Although this is adequate for establishing the nature of the instability, it leaves unanswered the question of the end state of the instability, i.e., at what amplitude the warp will saturate. In addition, there are a number of other complications which we have so far ignored, including torquing by a companion, torques due to X-ray heated winds (as considered by Schandl \& Meyer 1994), and opacity effects due to material intervening between the central radiation source and the disk, particularly the shadowing of the outer parts of the disk by the warp itself. It is also important to establish the observational consequences of radiation-driven warping; these will include not only the shape and precession rate of the disk, but the effect of the self-irradiation on the thermal state of the disk. Answering any of these questions will almost certainly require three-dimensional numerical simulations, but these are now well within reach of modern computing capabilities.

Acknowledgments. We thank Jim Pringle - the discover of the radiationdriven warp instability - for his constant encouragement and his active collaboration in some of the work described above. We also acknowledge support from NSF grants AST-9120599 and AST-9529175, and from NASA Long-Term Astrophysics Program grant NAGW-4454. Finally, we note with sorrow the recent passing of Jacobus Petterson, who pioneered the study of warped accretion disks.

\section{References}

Boynton, P.E., Crosa, L.M., \& Deeter, J.E. 1980, ApJ, 237, 169

Brecher, K. 1972, Nature, 239, 325

Chevalier, R.A. 1976, Astrophys. Letters, 18, 35

Cowley, A.P., et al. 1991, ApJ, 381, 526

Greenhill, L.J., Jiang, D.R., Moran, J.M., Reid, M.J., Lo, K.Y., \& Claussen, M.J. 1995, ApJ, 440, 619

Herrnstein, J., Greenhill, L., \& Moran, J. 1996, ApJ, 468, L17

Hut, P., \& van den Heuvel, E.P.J. 1981, A\&A, 94, 327

Iping, R.C., \& Petterson, J.A. 1990, A\&A, 239, 221 
Katz, J.I. 1973, Nature Phys. Sci., 246, 87

Lasota, J.P., Abramowicz, M.A., Chen, X., Krolik, J., Narayan, R., \& Yi, I. 1996, ApJ, 462, 162

Maloney, P.R., Begelman, M.C., \& Nowak, M.A. 1996, in preparation.

Maloney, P.R., Begelman, M.C., \& Pringle, J.E. 1996, ApJ, in press.

Maoz, E. 1995, ApJ, 447, L91

Margon, B. 1984, ARA\&A, 22, 507

Merritt, D., \& Petterson, J.A. 1980, ApJ, 236, 255

Miyoshi, M., Moran, J., Herrnstein, J., Greenhill, L., Nakai, N., Diamond, P., \& Inoue, M. 1995, Nature, 373, 127

Neufeld, D.A., \& Maloney, P.R. 1995, ApJ, 447, L19

Neufeld, D.A., Maloney, P.R., \& Conger, S. 1994, ApJ, 436, L127

Papaloizou, J.C.B., \& Pringle, J.E. 1982, MNRAS, 200, 49

Petterson, J.A. 1977, ApJ, 216, 827

Petterson, J.A., Rothschild, R.E., \& Gruber, D.E. 1991, ApJ, 378, 696

Priedhorsky, W.C., \& Holt, S.S. 1987, Space Sci. Rev., 45, 291

Pringle, J.E. 1992, MNRAS, 258, 811

Pringle, J.E. 1996, MNRAS, 281, 357

Roberts, W.J. 1974, ApJ, 187, 575

Schandl, S., \& Meyer, F. 1994, A\&A, 289, 149

Smale, A.P., \& Lochner, J.C. 1992, ApJ, 395, 582

Tananbaum, H., Gursky, H., Kellogg, E.M., Levinson, R., Schreier, E., \& Giacconi, R. 1972, ApJ, 174, L143

Trümper, J., Kahabka, P., Ögelman, H., Pietsch, W., \& Voges, W. 1986, ApJ, $300, \mathrm{~L} 63$

White, N.E., Nagase, F., \& Parmar, A.N. 1995, in X-Ray Binaries, ed. W.H.G. Lewin, J. van Paradijs, \& E.P.J. van den Heuvel (Cambridge: Cambridge University Press), p. 1 